\title{
Myasthenia Gravis Misdiagnosed as Post-Thyroidectomy Recurrent Laryngeal Nerve Palsy: A Case Report
}

\author{
Sripriya $R^{1}$, Reesha Joshi ${ }^{2 *}$, Sivashanmugam $T^{3}$, Ravishankar $M^{4}$ \\ ${ }^{1,3,4}$ Professor, Department of Anaesthesiology, Mahatma Gandhi Medical College and Research \\ Institute, Puducherry, ${ }^{2}$ Assistant Professor, Department of Anaesthesiology, Saveetha Medical \\ College \& Hospital, Tamil Nadu, India.
}

\begin{abstract}
About $85 \%$ of patients with myasthenia gravis present with ptosis and generalised muscle weakness, but $15 \%$ may present with pure bulbar symptoms which can be misdiagnosed. We describe a 60-year old female patient with multinodular goitre who had symptom of dysphagia and was subjected to total thyroidectomy. Post-operatively she developed respiratory distress and had difficulty in phonation. Bilateral vocal cord palsy was identified and emergency tracheostomy was done. Review of the patient revealed a mass in the anterior mediastinum and the patient showed improvement with pyridostigmine. Thymoma was confirmed in the computed tomography scan. This case highlights the unanticipated problems faced due to an undiagnosed myasthenia gravis and the importance of having a high index of suspicion of myasthenia gravis in patients with subtle bulbar symptoms.
\end{abstract}

Key-words: thyroid surgery, myasthenia gravis bulbar symptoms, myasthenia: muscle relaxant effects, myasthenia gravis: postoperative management.

\section{Introduction}

Myasthenia Gravis (MG) is a chronic autoimmune disease characterized by fatigable skeletal muscle weakness. About $85 \%$ of patients present with ptosis and generalised muscle weakness, but $15 \%$ may present with bulbar symptoms like dysphagia, dysphonia and dysarthria. ${ }^{1}$ This is seen especially with late ageonset MG and this group can be misdiagnosed. MG requires a tailored intra-operative management with caution towards muscle relaxants. $^{2}$

We present a rare case of undiagnosed bulbar MG who presented as a thyroid mass and had postoperative complications after surgery.

\section{Case Report}

A 60-year old female, weighing $55 \mathrm{~kg}$ was scheduled for total thyroidectomy for

$$
\begin{aligned}
& \text { *Correspondence: Reesha Joshi } \\
& \text { E mail: } \underline{\text { reesha.joshi@gmail.com }}
\end{aligned}
$$

\section{https://orcid.org/0000-0002-5674-2228}

Received: 03/09/2019

Accepted: 02/10/2020

DOI: http:/doi.org/ 10.4038/slja.v29i1.8680 multinodular goitre present since three years. She gave a history of dysphagia with solids for one month which was attributed to thyroid swelling increasing in size. Thyroid function tests were normal. Indirect laryngoscopy showed normal vocal cord (VC) movements. X-ray neck showed no tracheal compression or deviation (Figure 1a). General physical examination and investigations were normal.

During surgery induction, intubation, maintenance and intraoperative monitoring followed standard protocol. IV vecuronium $0.1 \mathrm{mg} / \mathrm{kg}$ was used. The surgery lasted 2.5 hours. The last dose of vecuronium $1 \mathrm{mg}$ was administered one hour before end of surgery. Neuromuscular blockade was reversed with 2.5 $\mathrm{mg}$ neostigmine and $0.5 \mathrm{mg}$ glycopyrrolate and patient extubated after ensuring adequate tidal volume (TV) and sustained handgrip. VC examined using a video laryngoscope was normal. She was shifted to post-anaesthesia care unit (PACU).

In PACU, she had difficulty breathing. Saturation dropped to $88 \%$ despite oxygen supplementation. She was conscious and responding to commands. There was generalized muscle weakness with motor power of $1 / 5$. After ventilatory support with Bain's circuit she became comfortable. Train-of-Four (TOF) showed only three twitches with fade. 
Suspecting residual neuromuscular blockade, neostigmine $1 \mathrm{mg}$ with glycopyrrolate $0.2 \mathrm{mg}$ was administered. Gradually muscle power improved to $3 / 5$ in 30 minutes, with an improvement of TV. After 45 minutes, the TOF ratio was 0.8 . Over the next 3 hours, motor power improved to $5 / 5$. She was shifted to ICU.

On POD-2 she was tachypnoeic, unable to vocalize or lie supine. Oxygen supplementation was increased to maintain $90 \%$ saturation. Breathing was paradoxical. ABG showed hypoxemia with mild respiratory alkalosis. Indirect laryngoscopy showed pooling of saliva in bilateral pyriform fossae, and sluggishly moving VC. Suspecting RLN injury an emergency tracheostomy was performed using IV propofol.

Post-tracheostomy, she was comfortable with pressure support of $5 \mathrm{cmH}_{2} \mathrm{O}$. The neurologist suspected MG based on findings of ptosis on prolonged gaze, absent gag reflex and inability to hold outstretched hand. Chest X-Ray was retaken. On the right heart border was a mass distinctly separate from the heart shadow (Figure 1b) probably a thymoma. All her
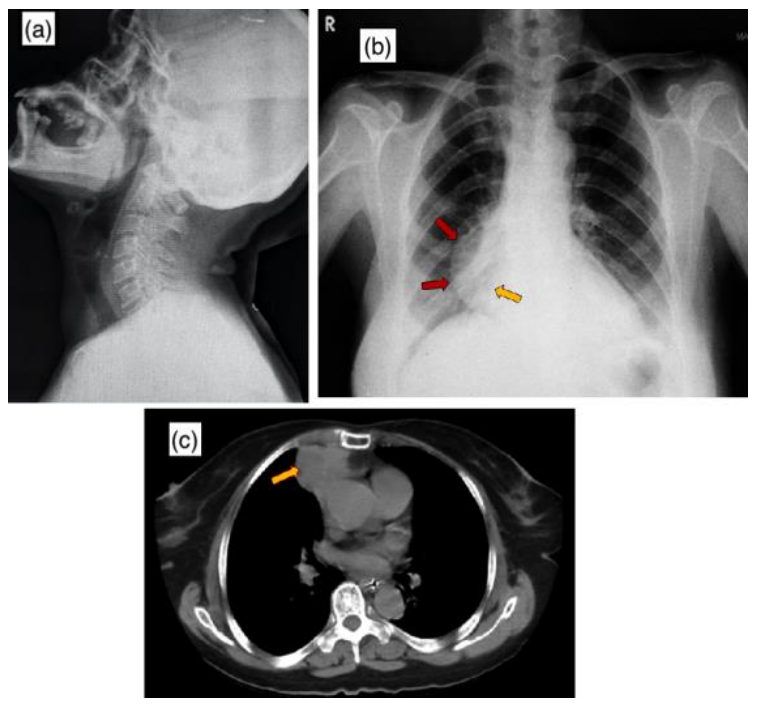

\section{Figure 1}

Radiological findings in the patient: (a) Preoperative X-ray soft tissue neck shows no tracheal compression in lateral view; (b) CXR in PA view. The yellow arrows indicate the right heart border while the red arrows indicate the thymoma; (c) The computed tomography scan shows an anterior mediastinal mass on the left side confirming a thymoma (arrow). symptoms could be attributed to MG. The stress of surgery had precipitated a myasthenic crisis. The patient improved after starting pyridostigmine $60 \mathrm{mg}$ three times a day. CT scan of thorax confirmed thymoma of size $4 \times 3 \times 5 \mathrm{~cm}$ (Figure 1c). The next day tracheostomy was decannulated. Thymectomy was scheduled for a later date.

\section{Discussion}

In this case, preoperatively patient had dysphagia to solids but we attributed it to thyroid mass. She developed neuromuscular weakness in the immediate post-operative period for which an additional dose of reversal was given. Had MG been diagnosed before surgery, drugs or thymectomy would have alleviated her symptoms. Had myasthenic crisis been diagnosed, tracheostomy could have been avoided.

The incidence of MG in India is only 2.1$5 / 100,000$ per year. ${ }^{3} \mathrm{We}$ hardly encounter patients with MG for incidental surgeries. A few cases have been reported where postoperative respiratory distress and muscle weakness have led to retrospective diagnosis of $\mathrm{MG.}^{4,5}$

Repetitive nerve stimulation (RNS) which shows a decrement in amplitude and area of the compound muscle action potentials (CMAP) and post-activation exhaustion in response to tetanic contraction confirms the diagnosis of MG. RNS however requires sophisticated equipment. ${ }^{6}$

The bedside neuromuscular monitors used to quantify the depth of muscle relaxation don't measure CMAP but interpret neuromuscular transmission based on the acceleration of a particular muscle to applied stimulus. ${ }^{6}$ TOF, which is the most frequently used function, shows fade both in MG (implying fatigability) as well as in inadequate reversal. The fade in TOF in our patient was wrongly attributed to inadequate reversal. Post-tetanic facilitation or post-tetanic exhaustion (by observing the response to a single twitch following tetanic stimulation) can differentiate the two conditions. In case of an inadequate reversal, the tetanic stimulus can increase the acetylcholine in the neuromuscular junction, causing a facilitated response, while in the case of MG, response to the twitch is diminished indicating post-tetanic exhaustion. 
Early versions of bedside neuromuscular monitors had the provision of delivering either a single twitch or tetanus. ${ }^{7}$ The tetanic stimulus was used to visually detect fade. With the introduction of TOF which could establish fade and PTC to quantify the depth during intense neuromuscular blockade, no further information was obtained from tetanic stimuli so it was taken off from most newer monitors. Hence posttetanic exhaustion essential for differentiating weakness due to MG from residual action of NDMR cannot be determined using these.

Retrospectively, we identified instances where MG could have been suspected and discuss it for use in trouble-shooting similar situations:

1. We need to have a high index of suspicion of MG in patients with bulbar symptoms.

2. The well-circumscribed homogenous opacity in CXR was mistakenly considered as the right heart border. If observed closely, the cardiac silhouette can be identified as separate from the mass (Fig. 1b). Although thymoma can occur at several locations, the anterior mediastinum is most common. ${ }^{8}$

3. In unexpected delay in recovery from NDMR, it is best to first rule out neuromuscular disorders before considering myopathies. Myasthenia is responsible for $75 \%$ of neuromuscular disorders. ${ }^{9}$

4. Although ptosis was not evident in the postoperative period, it needs to be elicited on prolonged gaze. ${ }^{10}$

This case report highlights the importance of having a high index of suspicion of myasthenia gravis in patients with bulbar symptoms. In undiagnosed cases, neuromuscular problems should be considered while troubleshooting for prolonged neuromuscular weakness in patients with normal hepatic and renal functions.

\section{References}

1. Oosterhuis $\mathrm{H}$ J. The natural course of myasthenia gravis: A long term follow up study. J Neurol Neurosurg Psychiatry 1989; 52:

1121-7

https://doi.org/10.1136/jnnp.52.10.1121

PMID: 2795037

2. Aarli JA. Late-onset myasthenia gravis: a changing scene. Arch Neurol. 1999; 56: 25-7 https://doi.org/10.1001/archneur.56.1.25 PMID: 9923757

3. Murthy JMK. Thymectomy in myasthenia gravis. Neurol India. 2009; 57: 363 https://doi.org/10.4103/0028-3886.55567 PMID: 19770532

4. Dunsire MF, Clarke SG, Stedmon JJ. Undiagnosed myasthenia gravis unmasked by neuromuscular blockade. Br J Anaesth. 2001; 86:7 27-30

https://doi.org/10.1093/bja/86.5.727

PMID: 11575353

5. Ozel F, Aydin Altunkan A, Azizoglu M. Postoperative Respiratory Failure in a Patient with Undiagnosed Myasthenia Gravis. Turk J Anesth Reanim. 2016; 44: 108-10 https://doi.org/10.5152/TJAR.2016.03274 PMID: 27366570

6. Lo YL, Dan YF, LeohTh, Tan YE, Ratnagopal $P$. Decrement in area of muscle responses to repetitive nerve stimulation. Muscle Nerve. 2003; 27: 494-6

https://doi.org/10.1002/mus.10339

PMID: 12661052

7. Christie TH, Churchill-Davidson HC. The St. Thomas's Hospital nerve stimulator in the diagnosis of prolonged apnoea. Lancet. 1958; 1: 776

https://doi.org/10.1016/s0140-6736(58)91583-6 PMID: 13526270

8. Ellis K, Austin J, Jaretzki A. Radiologic detection of thymoma in patients with myasthenia gravis. Am J Roentgenol 1988; 151: 873-81

https://doi.org/10.2214/ajr.151.5.873

PMID: 3263017

9. Vincent A1, Palace J, Hilton-Jones D. Myasthenia gravis. Lancet 2001; 357: 2122-8 https://doi.org/10.1016/S0140-6736(00)05186-2 PMID: 11445126

10. Pasnoor M, Dimachkie MM, Farmakidis C, Barohn RJ. Diagnosis of Myasthenia Gravis. Neurol Clin 2018; 36: 261-74 https://doi.org/10.1016/j.ncl.2018.01.010 PMID: 29655449 